\title{
A review on implementation of electronic health in iran literature
}

\begin{abstract}
Introduction: The important role of electronic health as well as the importance of health care systems may lead to the essential infrastructures for electronic health especially in developing countries. Iran is a developing country that has national plans such as TAKFA and SEPAS as well as organizational e-health projects such as HISs in hospitals. The present study is a non-systematized review conducted in order to investigate literature about electronic health in Iran.
\end{abstract}

Methods: PubMed, Google Scholar, Science direct, Magiran, SID and Irandac were searched for "Telemedicine", "E-health" and "Iran" keywords from Sep 2005 to Apr 2016. About 74 articles were found which were evaluated and finally 40 of them were selected based on their relevance to the study.

Results: Research findings showed these subjects: E-health readiness factors, types of telemedicine implementation, barriers and benefits of e-health in Iran. Some types of e-health were: Text messaging, telephone support or follow- up; and web-based interventions were effective channels of communication for health care and diseases management. Clinical librarians were able to provide reliable information to remote and rural physicians, the fact which was also verified by the specialists. Although literatures showed that E-health was beneficial for healthcare system, but there were managerial, technical, social and organizational barriers, which have to be moderated.

Conclusion: Similar to other countries, implementation of e-health in Iran is subjected to challenges. Effective factors for E-health implementation are: efficient administration and behavior intention in E-health adoption. Hence, E-health providers need to adhere to the proposed solution in order to overcome the mentioned challenges in such complicated projects.

Keywords: e-health, telemedicine, Iran
Volume 5 Issue 5 - 2017

\author{
Asieh Darvish,' Mitra Arjmandi Far² \\ 'PhD Candidate in Information Technology Management, School \\ of Nursing and Midwifery, Iran \\ ${ }^{2} \mathrm{PhD}$ student of Reproductive Health, School of Nursing and \\ Midwifery, Iran
}

Correspondence: Mitra Arjmandi Far, PhD student of Reproductive Health, School of Nursing and Midwifery, Iran Tehran Tohid Square-School of Nursing and Midwifery, Tehran University of Medical Sciences, Tehran, Iran, Tel 09122841970, Emailarj7087@yahoo.com

Received: July 20, 2017| Published: August 10, 2017

\section{Introduction}

Information technology has had a profound impact on various fields. Health care institutions are not exempted from this effect. These institutions generate a high volume of information which should be collected, distributed, registered, retrieved and summarized. The application of technology in health care in forms of electronic health record and e-health system is one of the most important issues which can be helpful in improvement of health care quality. ${ }^{1}$ One of the first steps could be providing the hardware, national standardization of protocols and improvement of computer skills of caregivers. $^{2-4}$ Supports and contribution of different ministries and non-governmental organizations are essential in effective e-health implementation. ${ }^{5}$ Various e-health applications such as telemedicine, email consultation and distance education have been performed in strategic programs of different countries. ${ }^{6}$ Implementation and practice of these applications have received higher attention in some countries. World Health Organization (WHO), expressing the benefits of e-health, has announced that e-health must be one of the essential parts of any strategy and the most important plan in order to vicissitude the health care system in the $21^{\text {st }}$ century.

The structural characteristics of Iran's health system reminding the necessity of information technology development in medical field are as follows: variation of disease in reprobated areas and concentration of resources in large cities, dramatic effect of awareness in improving health and reducing the disease incidence, the necessity of immediate access to patients' health records by physician, access to information and disease statistics in medical research, medical cooperation as the essential requirement for synergy, the need of professional counseling without the physical presence of patients, the necessity of increasing treatment efficiency, patients' reverence and providing the best service with the least concern for patients and relatives and also providing the necessary equipment for physician. ${ }^{1}$ It seems more attention is required to present the strategies which increase the probability of success in implementation of any practical means of this technology in Iran. E-health has gradually stepped into international health system to increase the quality of treatment and access to economic treatment. The increasing demand for education and research has made e-therapy the only solution to these challenges. ${ }^{7,8}$ The present study is a nonsystematized review to investigate literature about electronic health in Iran.

\section{Method}

All the studies considering Electronic Health in Iran were identified by searching electronic databases such as PUBMED, Google Scholar, Science direct Magiran, SID and Irandac. The keywords used for search included Telemedicine, E-health and Iran. All qualitative and quantitative studies published from 2005 to 2016 investigating any E-health issues in Iran were selected if included an appropriate sample and presented valid results. Only the articles' full texts and 
English language were used. About 74 articles were available which were evaluated and finally 40 of them were selected based on their relevance to the study.

\section{Results}

\section{Literature about telephone or mobile health monitoring in Iran}

In our search, 11 articles addressed the effect of cell phone text messaging, ${ }^{9,10}$ telephone follow-up, ${ }^{11,12}$ telephone support or screening ${ }^{13,14}$ and home based health care systems ${ }^{15}$ on health. Some articles were about, e-health model using mobile agents, ${ }^{16}$ software program that can receive the ECG signal and real-time processing in the mobile phone and then transferring it to a remote medical center, ${ }^{17}$ awareness of medical students in clinical stage about mobile health technology application,,$^{18}$ diagnosis of Parkinson's disease in human using voice signals. ${ }^{19}$

\section{E-health and telemedicine implementation in Iran}

There were 13 articles about implementation of e-health and telemedicine in Iran: readiness assessment for e-health records implementation, ${ }^{20}$ telemedicine operational in selected hospital of Isfahan University of Medical Sciences in 2014, ${ }^{21}$ the effect of e-health expenditure on economic growth in Iran, ${ }^{22}$ effective elements on e-health deployment in Iran, ${ }^{23}$ key aspects of providing healthcare services in disaster response stage, ${ }^{24}$ electronic health in perspective of healthcare managers in south of Iran, ${ }^{25}$ e-health readiness assessment framework on Iran, ${ }^{26}$ determining the awareness and attitude of employee in deputy of health in Isfahan university of medical science toward telemedicine and its advantage, ${ }^{27}$ technological feasibility of a nursing clinical information system, ${ }^{28}$ medical information in Iran, ${ }^{8}$ factors affecting electronic medical record acceptance by specialist physicians, ${ }^{29}$ e-health implementation challenges in Iranian medical centers $^{30}$ and chances and challenges of telemedicine in Iran. ${ }^{31}$

\section{Web based training or interactive in Iran}

The studies in this field included comparing the effect of traditional, web based and simulation training on midwifery student, clinical competence in postpartum hemorrhage management, ${ }^{32}$ a web-based interactive lifestyle modification program improves lipid profile and serum adiponectin concentrations in patients with metabolic syndrome, ${ }^{33}$ and a web based study about health-related quality of life among people participating in a metabolic syndrome e-screening program. ${ }^{34}$ There were three articles in our search which was about HIS and one article about Information and Communication Technologies (ICTS): ${ }^{35}$ The status of hospital information systems in Iranian hospitals.$^{36}$ HIS in Iranian military hospitals, ${ }^{37}$ a case study about evaluation of hospital information systems in Iran. ${ }^{38}$

\section{Discussion}

The findings of Maslakpak $\mathrm{H}$ et al., ${ }^{9}$ demonstrated that training and distance-monitoring via SMS and reminder cards promote medication adherence of patients (48). Karimi Rouzbahani recorded and processed voice signals and provided different aspects of the diagnosis of parkinson's disease, from which the physicians were able to choose the one with higher diagnostic accuracy. ${ }^{19}$ Soleimanian et al., ${ }^{15}$ evaluated four home-based e-health care systems including telemonitoring of patients system, home care system, monitoring Alzheimer patient system and heart failure management system. All of them were implemented in the internet based platforms within emphatic on alarm and home medical care. They also present noncritical systems like home based e-health care systems and explained the functional plans which have been performed in the past years in Iran such as electronic prescription, taking online visit time, electronic health records in hospitals and many more. These technologies facilitates convenient and loyal communications between patient and medical staff, as well as the medical, imaging and health data transformations to clients and cooperation with all of applied agents. ${ }^{15}$

Faghanipour et al., ${ }^{13}$ suggested cell phone as a useful tool for helping individuals to lose weight. ${ }^{13}$ The findings of Hemmati Maslakpak, research demonstrated that training and distancemonitoring via SMS and reminder cards promote medication adherence among hypertension patients. ${ }^{9}$ In Pouyan paper, a 3-layer agent-based model was proposed which is supposed to be used as a general framework for e-health systems to facilitate health care processes. This 3-layer model consisted of patient, clinic and centralhospital layers. These layers are hierarchically and horizontally related to each other. This relationship is defined to achieve more accuracy in treatment recognition, quick treatment, and diagnosis. It facilitates the relationship between different parts of e-health procedures and reduces the workload and complexity of linking different interfaces. Mobile Agents technology is used to distribute the processing load as well as to support a more flexible peer-to-peer model, scalability and decentralization of control. ${ }^{16}$

In the study conducted by Ehteshami, the highest percentage of awareness about mobile health technology among medical students in the clinical stage of Azzahra educational hospital is 45.6 in nature fields, and their lowest percentage of awareness is 17.8 in the infrastructure domain. In addition, their mean awareness value about all areas is 54.4. The highest percentage of using mobile health technology by medical students is 14.6 in the education area, and their lowest percentage of usage is 6.8 in the treatment area. Their mean usage of all areas is 9.4 as well. ${ }^{18}$ Kooki RB et al. ${ }^{17}$ designed a software program to receive the ECG signal, its real-time processing by mobile phone and then transfer it to a remote medical center. Heart rate is a vital signal to determine a patient's health status. With continuous measurement, they can be successful in early and rapid diagnosis of two major adverse cardiac disorder such as bradycardia (decreases in heart rate) and tachycardia (increase in heart rate). Primary experiments had successful results for their health care system. The patient can install the program on his/her mobile phone (windows mobile) and his/her heart condition would be controlled all day long. ${ }^{17}$ The results of this study ${ }^{11}$ proved that using telenursing for follow-ups has positive effects on metabolism control and $\mathrm{HbA1C}$ and can improve it in 12 weeks. The results also showed that phone follow-ups can improve the process of self-care and the control of glycemic index in patients with type II diabetes. ${ }^{11}$ Also, Roozbahani $\mathrm{K}$ et al., ${ }^{12}$ demonstrated that telephone follow-up could significantly reduce fasting blood glucose levels in mothers with gestational diabetes and also increased the rate of postpartum screening test. ${ }^{12}$ In a study performed by Vakili et al. the findings demonstrated that using mobile phone short messaging system can improve the healthy food choices regarding Vitamin A rich fruits and vegetables and fish among postmenopausal women..$^{10}$ On the other hands Mohammad-Alizadeh et al. ${ }^{14}$ could not provide evidence to show that telephone support of a midwife during postpartum period could have a preventive effect on postpartum depression. ${ }^{14}$ 
Saghaeiannejad et al., ${ }^{20}$ investigated telemedicine operational feasibility in the selected hospitals of Isfahan University of Medical Sciences in 2014. Telemedicine operational feasibility was positive and managers and most of the doctors believed that the proposed system is able to resolve many work challenges. Investigating technological infrastructure problems, internet speed, and in general telemedicine technical feasibility will lead to propose a system, which is consistent with existing facilities. Investment- in strengthening cultural infrastructure will be effective in the success of system implementation. ${ }^{21}$ Sadr and Gudarzi Farahani analyzed the economic effect of e-Health and also its influences on economic growth in Iran regarding the annual data covering the period of 1970-2011. For this purpose vector autoregressive model (VAR) was used. First, stability of variables was examined by the use of Dickey-Fuller test. Next, analysis of Johnson test was employed for considering the convergence among six variables. The results showed that variables such as e-health expenditure to GDP ratio and the ratio of investment to GDP and Growth rate of graduates have positive effect on growth rate. Also population growth variable has a negative effect on growth rate. ${ }^{22}$ Keshvari et al., ${ }^{27}$ used a descriptive - analytical method with sectional information gathering. The investigated population consisted of all managers and experts employed by Deputy of health of Isfahan University of Medical Science.

The sample size was determined based on inclusion criteria to be 60 people. The data collection tool was a questionnaire designed by the researcher in order to determine the awareness and attitude of the subjects. Research findings showed that the awareness and attitude of managers and experts toward telemedicine was mediocre. Spearman correlation test showed that there is a correlation between the educational degree and awareness of the subject, however the correlation coefficient was lower than 0.5 which shows a weak correlation (0.451). On the other hand, the calculated P-value of 0.008 showed that there is a significant relationship between the education and awareness of managers regarding telemedicine. ${ }^{27}$ Jeddi $\mathrm{R}$ et al., ${ }^{28}$ conducted a cross-sectional study in three steps. The aim of this study was to determine technical feasibility of a nursing clinical information system (NCIS) in Mazandaran province, Iran, 2015. In the first step, a data collection tool was developed through an unsystematic literature review. In the second step, a questionnaire was developed and validity of the tool was confirmed by receiving opinions of faculty members and calculating indices of Content Validity Index (CVI) and Content Validity Ratio (CVR). The questionnaire reliability was confirmed by calculating Cronbach's alpha coefficient $(\alpha=0.72)$. In the third step, the feasibility of NCIS implementation was evaluated by forming a panel of IT experts $(n=30)$, and a questionnaire. Data were collected by 5 -point Likert scale varying from very low to very high (scoring 1-5). Scores of each item were calculated and score percentage was determined. Chi-square and Fisher Exact tests were also used.

Maximum possibility of implementing NCIS was in the hardware area, additional equipment $(92.6 \%)$, in the area of software, financial software $(99.4 \%)$, in the area of network equipment, the possibility of integration with other internal systems, $(92.6 \%)$ and in the area of network security, the possibility of backup version for security purposes $(97.4 \%)$. Type of employment was statistically significant according to IT experts' opinions $(p=0.014){ }^{28}$ In Sharifi's study, a panel of experts from Isfahan, Iran, who specialized in e-health applications, was selected for a qualitative survey. Results showed efficient administration of e-health implementation improves the quality of healthcare, reduces costs and medical errors, and enables transfer of healthcare resources to rural areas, among other benefits. However, there are numerous issues affecting the successful implementation of e-health such as lack of standardized e-health applications, deployment costs, training costs, legal challenges, privacy and security fears, implementation and acceptance time of such applications, technical difficulties, educational issues, resistance to change, and pilot projects or small-scale implementations with limited numbers of patients in healthcare organizations. ${ }^{30}$

Salehahmadi and Hajialiasghari conducted a survey in Tehran University of Medical Sciences in Iran. Between May $14^{\text {th }}$ and August $14^{\text {th }}$ 2012, 90 male medical specialists and 42 female ones from different hospitals of Tehran Medical Sciences University, were enrolled by a simple random sampling method. They all completed a questionnaire to consider what are telemedicine chances and challenges. Making use of telemedicine was a profitable alternative in remote, rural/urban places especially in new project of "family physician" presented by Iran Ministry of Health. The results also highlighted that security considerations was an inevitable challenge of telemedicine, while shorter hospital stays and reduces warm ischemic time, and reduced morbidity and mortality rates were telemedicine merits. Despite the results of majority of previous studies, telemedicine has been recognized as a cost-effective alternative. Cultural, language distinctions as well as the level of literacy were barriers on deploying telemedicine. There was no strong evidence showing that using telemedicine caused a decrease in tactile feedback. ${ }^{31}$

Jahanbakhsh et al. performed a research in which a number of Hospitals in Isfahan Iran were selected using convenient sampling. E-health research professionals went there to observe their HIS and collect required data as a qualitative survey. The design of interview questions was based on the researchers' experiences and knowledge in this area along with elementary interviews with experts on HIS utilization in hospitals. Efficient administration of e-health implementation improves the quality of healthcare, reduces costs and medical errors and makes healthcare resources available to rural areas, etc. However, there are numerous issues affecting the successful utilization of e-health in hospitals, such as a lack of a perfect HIS implementation plan and well-defined strategy, inadequate ITsecurity for the protection of e-health-related data, improper training and educational issues, legal challenges, privacy concerns, improper documentation of lessons learned, resistance to the application of new technologies, and finally lack of recovery plan and disaster management. These results along with some informative stories were extracted from interview sessions to uncover associated challenges of HIS utilization in Iranian hospitals. ${ }^{10}$ Farahani et al..$^{38}$ attempted to assess the maturity and applicability of HISs in Iranian hospitals. Two Iranian military hospitals were selected for HIS maturity assessment. Data were collected through interviewing related experts and the use of some software and documentation analysis. Then the data were compared with the Electronic Medical Record Adoption Model (EMRAM).

The results revealed that the HISs in these military hospitals had reached different stages of EMRAM while they could reach upper HIS maturity stages. ${ }^{22}$ Sadr in his paper presented e-health solutions and ambient assisted living systems developed by researchers of the Faculty of Management and Economics University in Allame Tabatabae. He also touched the issue of interoperability that is crucial for correct interconnection of medical and other devices and information system she proposed a high level functional architecture 
of a system that can satisfy the requirements of interoperability. In this paper he had tried to present several ICT solutions directly applicable to healthcare domain, either as part of information systems, systems supporting rescue and logistic operations, assistive systems, or home care support systems.

The most challenging tasks are integration tasks for applications, such as management and logistics in case of natural disaster; organization of help, multilingual support in information systems. He can utilize results from planning and scheduling systems, coalition formation, distributed decision support and use of mobile technologies. In addition, several presented solutions have been already introduced to routine practice. Solutions developed in EU projects were successfully verified in pilot tests. Presented projects showed that successful applications need coherent approach of experts from many different disciplines, i.e. information technology, electronics, communication technology, medicine. Standardization can make the way from an idea to an application much easier and faster. Thus acceleration of standardization process represents a key issue. It is important that involved companies, researchers, and standardization bodies agree and cooperate towards the ultimate goal - defined standards. There already exists one example of successful agreement on defined standard DICOM. DICOM is now widely accepted as a standard for medical imaging data and nobody uses another data format. Similarly we should reach such agreement for other data types. ${ }^{34}$

Fakari $\mathrm{R}$ et al., ${ }^{32}$ performed a study to compare the effect of traditional, web based and simulation training on clinical competencies of midwifery students in postpartum hemorrhage (PPH) management. This semi-experimental study was performed among 91 midwifery students in Mashhad School of Nursing and Midwifery, 2013. No significant difference was found between the three groups in clinical competence to manage PPH before the intervention $(\mathrm{P}>0.326)$. One week after training, the mean score of students' clinical competence showed significant increase in all groups $(\mathrm{P}<0.001)$. The intergroup comparison indicated no significant difference between the groups in clinical competence one week after the intervention $(\mathrm{P}=0.128) .{ }^{32}$ Farhangi A et al., ${ }^{33}$ investigated the effect of a 6-month web-based interactive lifestyle modification program on anthropometric variables and biochemical risk factors of cardiovascular disease. The study had been carried out among 160 patients with metabolic syndrome (intervention, $\mathrm{n}=80$; control, $\mathrm{n}=80$ ). The mentioned study showed that a web-based intervention was effective in weight loss and improving cardio-metabolic factors in patients with metabolic syndrome after a 6-month intervention. ${ }^{33}$ Jahangiry et al. performed a web-based, crosssectional study. Participants were recruited through online registering on the study website. Then, those who met the study criteria (waist circumference $[\mathrm{WC}] \geq 90$ and blood pressure $[\mathrm{BP}] \geq 130 / 85$ ) were contacted and invited for the clinical assessments, if they wish. Baseline measurements were MetS (Metabolic Syndrome) risk factors (weight, WC, body mass index and BP, total cholesterol, lowdensity lipoprotein-cholesterol, high-density lipoprotein-cholesterol, triglycerides, and fasting blood glucose) and health-related QOL (HRQOL) that was measured using the short form-36 (SF-36). People with MetS experienced lower HRQOL than those lacking MetS. Internet as a powerful medium offers a novel setting for delivery health information. It seems that high BP and abdominal obesity are associated with lower HRQOL in the participants with MetS. A web-based prevention program could make people aware for their vulnerability to MetS and its consequent complications. ${ }^{37}$

\section{Conclusion}

The major obstacles to establish e-health in Iran are lack of a specific strategy, ambiguous and complicated information technology infrastructure, two cultural and educational problems in relation to training and practice in information technology and communication skills, rapid changes of managers especially in Ministry of Health and Welfare, inability to attract skilled information technology technicians in e-health field, unclear mechanism to fund e-health system, lack of codified technical standards and weak health service implementation methods. Infrastructure of information and communication technology in Iran is complex and ambiguous and e-health strategies and priorities have not been determined yet. The ability to develop EHR and an electronic health record for every Iranian can be reached out in near future. Therefore, with respect to the importance and necessity of flexible technological infrastructure for the integration of information between the health care centers and the necessity of a national health information network for the continuity of all centers involved in the public health, it is recommended that all contributors and policy makers in health field work collaboratively to eliminate the barriers and deficiencies to achieve to the desired objective within the prescribed time.

\section{Acknowledgements}

None.

\section{Conflict of interest}

The author declares no conflict of interest.

\section{References}

1. Farahmand F, Ahmadzade F. Need to Use Health Information Technology in Iran's Health System. Shiraz: College of Information and Management; 2007.

2. Tran VA, Seldon HL, Chu H, et al. Electronic healthcare communications in Vietnam in 2004. Int J Med Inf. 2006;75:764-770.

3. Kluge EH. Secure e-Health: Managing risks to patient health data. Int $J$ Med Inform. 2007;76(5-6):402-406.

4. Yu P, Li H, Gagnon MP. Health IT acceptance factors in longterm care facilities: A cross-sectional survey. Int J Med Inform. 2009;78(4):219-229.

5. Moisil I, Jitaru E. E-health progresses in Romania. Int $J$ Med Inform. 2006; 75(3-4):315-321

6. Eysenbach G. What is e-health? J Med Internet Res. 2001;3(2):20

7. World health organization regional office for the eastern mediterranean. What is e-Health? 2008.

8. http://www.unicef.org/iran/media 4101.htm,http://www.scict.ir/portal/ Home/, http://en.wikipedia.org/wiki/Health_care_in_Iran, http://www. who.int/en/,http://ispub.com/IJMI/4/1/4627\#

9. Masumeh MH, Safaie M. A Comparison between the effectiveness of short message service and reminder cards regarding medication adherence in patients with hypertension: a randomized controlled clinical trial. IJCBNM. 2016;4(3):209-218.

10. Vakili M, Abedi P, Afshari P, et al. The effect of mobile phone short messaging system on healthy food choices amongIranian postmenopausal women. J Midlife Health. 2015;6(4):154-159.

11. Lashkari T, Borhani F, Sabzevari S, et al. Effect of telenursing (telephone follow-up) on glycemic control and body mass index (BMI) of type 2 
diabetes patients. Iran J Nurs Midwifery Res. 2013;18(6):451-456.

12. Khorshidi Roozbahani R, Geranmayeh M, Hantoushzadeh S, et al. Effects of telephone follow-up on blood glucose levels and postpartum screening in mothers with gestational diabetes mellitus. Med J Islam Repub Iran. 2015;29:249.

13. Faghanipour S, Hajikazemi E, Nikpour S, et al. Mobile phone short message service (SMS) for weight management in iranian overweight and obese women: a pilot study. Int J Telemed Appl. 2013;785654.

14. Mohammad-Alizadeh-Charandabi S, Malakoti J, Sohrabi F, et al. The effect of telephone support on postpartum depression: a randomized controlled trial. J Caring Sci. 2013;2(2):147-155.

15. Abbasi Khalifehlou Z, Gharehchopogh FS. Comparison of pervasive home based health care systems under e-health perspectives. International Journal of Electronics, Electrical and Computational System IJEECS 2014;2(1):19-24.

16. Ali A Pouyan, Sadegh Ecrami, Momeneh Taban. A Distributed E-health Model Using Mobile Agents. The Seventh International Conference on Autonomic and Autonomous Systems. ICAS; 2011

17. Raeiatibanadkooki M, Quachani SR, Khalilzade M, et al. Real time processing and transferring ECG signal by a mobile phone. Acta Inform Med. 2014;22(6):389-392

18. Ehteshami A, Hachesu PR, Esfahani MK, et al. Awareness and using of medical students about mobile health technology in clinical areas. Acta Inform Med. 2013;21(2):109-112.

19. Rouzbahani HK, Reza Daliri M. Diagnosis of Parkinson's disease in human using voice signals. Basic and Clinical. 2011;2(3):12-20.

20. Ajami S, Ketabi S, Isfahani SS, et al. Readiness Assessment of Electronic Health Records Implementation. Acta Inform Med. 2011;19(4):224-227.

21. Saghaeiannejad-Isfahany S, Jahanbakhsh M, Shayan A. Telemedicine operational feasibility in selected hospitals of Isfahan university of medical sciences in 1393. International Journal of Health System and Disaster Management. 2015;3(4):189-194.

22. Sadr, Mohammad Hossein S, Farahani G, et al. Consideration the effect of e-health expenditure on economic growth in iran with VAR approach. Asian Journal of Business and Management Sciences. 2011;2(3):35-42.

23. Nasiripour AA, Rahmani H, Radfar R, et al. Effective elements on e-health deployment in Iran. African Journal of Business Management. 2012;6(16):5543-5550.

24. Pourhosseini SS, Ardalan A, Mehrolhassani MH. Key aspects of providing healthcare services in disaster response stage. Iran $J$ Public Health. 2015;44(1):111-118.

25. Bastani P, Abolhasani N, Shaarbafchizadeh N. Electronic health in perspective of healthcare managers: a qualitative study in south of Iran Iranian J Publ Health. 2014;43(6):809-820.
26. Rezai-Rad M, Vaezi R, Nattagh F. E-Health readiness assessment framework in Iran. Iranian J Publ Health. 2012;41(10):43-51.

27. Keshvari H, Haddadpoor A, Taheri B, et al. Determining the awareness and attitude of employees in deputy of health of isfahan university of medical science toward telemedicine and its advantages. Acta Inform Med. 2015;23(2):97-101.

28. Jeddi FR, Hajbaghery MA, Akbari H, et al. Technological feasibility of a nursing clinical information system. Electronic Physician. 2016;8(9):29422949.

29. Lakbala P, Mahboobeh 1, Inaloo KD. Factors affecting electroic medical record acceptance by specialist physicians. Lectre Notes on Iformation theory. 2014;2(4):316-321.

30. Sharifi M, Ayat M, Jahanbakhsh M, et al. E-health iplementation challenge in iranian medical center: a qualitative study in Iran. TMJ. 2013;19(2):122128.

31. Salehahmadi Z, Hajialiasghari F. Telemedicine in iran: chances and challenges. JPS. 2013;2(1):18-25.

32. Rashidi Fakeri F, Masoomeh Kord, Mazom SR, et al. Compairing Effect of Traditional, Web based and Simulation Training on Midwifery Students Clinical Competence in Postpartum Hemorrage Management. J azandaran Univ Med Sci. 2015;25(123):65-77.

33. Abbasalizad Farhangi M, Leila Jahangiry, Mirinazhad MM, et al. A webbased interactive lifestyle modification program improves lipid profile and serum adiponectin concentrations in patients with metabolic syndrome: the “Red Ruby" study. Int J Diabetes Dev Ctries. 2015;37(1):21-30

34. Jahangiry L, Shojaeezadeh D, Montazeri A, et al. Health-related quality of life among people participating in a metabolic syndrome e-screening program: A Web-based Study. Int J Prev Med. 2016;7:27.

35. Sadr, Mohammad Hossein S. Consideration the relationship between ICT and Ehealth. Journal of Biology, Agriculture and Healthcare. 2012;2(8):49-59.

36. Jahanbakhsh M, Sharifi M, Ayat M. The status of hospital information systems in iranian hospitals. Acta Inform Med. 2014;22(4):268-275.

37. Hekmat NS, Dehnavieh R, Behmard T, et al. Evaluation of hospital information systems in iran: a case study in the kerman province. Global Journal of Health Science. 2016;8(12):95-103.

38. Farahani RM, Sharifi M, Ayat M, et al. Hospital information system in iranian militry hospitals: a multiple case analysis. $J$ Arch Mil Med. 2004;2(4):e22853. 